\title{
EHMTI-0157. Specific strength training compared with interdisciplinary counselling for girls with tension-type headache - a randomised controlled trial
}

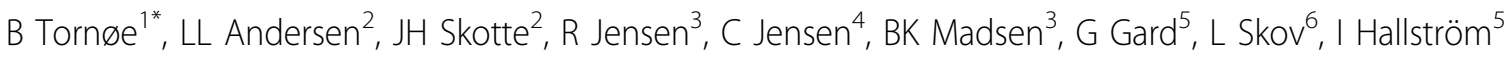 \\ From 4th European Headache and Migraine Trust International Congress: EHMTIC 2014 \\ Copenhagen, Denmark. 18-21 September 2014
}

\section{Background}

Childhood tension-type headache (TTH) is a prevalent and debilitating condition for the child and family. Low-cost non-pharmacological treatments are usually the first choice of professionals and parents. The study examined the outcomes of specific strength training for girls with TTH.

\section{Methods}

Forty-nine girls 9-18 years with TTH were randomised to patient education programmes with 10 weeks of strength training compared with counselling by nurse and physiotherapist. Primary outcomes were headache frequency, intensity and duration; secondary were neckshoulder muscle strength, aerobic power and pericranial tenderness, measured baseline, after intervention and at 12 weeks follow-up. HRQOL questionnaires were assessed at baseline.

\section{Results}

For both groups headache frequency decreased significantly, $\mathrm{p}=0.001$ and likewise for duration $\mathrm{p}=0.022$, with no significant between-group differences. The odds of having headache a random day decreased during 22 weeks by 0.65 (0.50-0.84) (OR (95\%CI)). For both groups neck extension strength decreased significantly with a decrease in cervicothoracic ratio to 1.7 , indicating a change in muscle balance. In the training group shoulder strength increased $\geq 10 \%$ in $5 / 20$ girls and estimated VO2 max increased $\geq 15 \%$ for $4 / 20$ girls.

\section{Conclusions}

Both training and counselling lead to headache reduction. Adjusting muscle-balance seems to precede strength gains. Exercising might lead to important changes in the child's physical capability and health. Restructuring patient education and examining dose-response of exercising is recommended.

No conflict of interest.

\section{Authors' details}

${ }^{1}$ Pediatric department, Herlev Hospital, Copenhagen, Denmark. ${ }^{2}$ Working environment, National Research Centre for the Working Environment, Copenhagen, Denmark. ${ }^{3}$ Department of Neurology Glostrup hospital University of Copenhagen, Danish Headache Centre, Copenhagen, Denmark. ${ }^{4}$ Aps, Huge Consulting, 4690 Haslev, Denmark. ${ }^{5}$ Faculty of Medicine, Department of Health Sciences, Lund, Sweden. ${ }^{6}$ Pediatric department, Herlev Hospital University of Copenhagen, Herlev, Denmark.

Published: 18 September 2014

\section{doi:10.1186/1129-2377-15-S1-E37}

Cite this article as: Tornøe et al:. EHMTI-0157. Specific strength training compared with interdisciplinary counselling for girls with tension-type headache - a randomised controlled trial. The Journal of Headache and Pain 2014 15(Suppl 1):E37. 